\title{
Trajectory forms as information for visual event recognition: $3-D$ perspectives on path shape and speed profile
}

\author{
EMILY A. WiCKELGREN \\ California State University, Sacramento, California \\ AND \\ GeOFFrey P. Bingham \\ Indiana University, Bloomington, Indiana
}

\begin{abstract}
Trajectory forms in events consist of the path shape and the speed profile (Bingham, 1987, 1995). Wickelgren and Bingham (2004) showed that adults can use the speed profile as visual information to recognize events from different perspectives, despite perspective distortions and differences in optical components. We now investigate whether adults can use trajectory forms to recognize events when the forms are viewed from 3-D perspectives and the path shape and speed profile vary. In Experiment 1, we tested recognition of events that differ in path shape (with the speed profile held constant). In Experiment 2, we tested recognition of events in which speed profiles were mapped onto circular paths. In Experiment 3, as a strong test of sensitivity to trajectory forms, we tested simultaneous separate recognition of speed profile and path shape when both varied across events. In all three experiments, events were viewed from multiple 3-D perspectives. The results show that both the shape of the path and the speed profile provide information for visual event recognition. We found that adults exhibit constancy (or view invariance) in being able to use trajectory forms to identify the same events when viewed from different 3-D perspectives.
\end{abstract}

Visual event perception is the process and ability of identifying events on the basis of information in the optic flow pattern. The earliest studies of information in visual event perception focused on the phase relations among the visible points in rhythmic or oscillatory events (Johansson, 1950). The role of relative phase has been studied extensively both in visual event perception studies (Bertenthal, 1996; Bertenthal \& Pinto, 1993, 1994; Bertenthal, Proffitt, \& Cutting, 1984; Bertenthal, Proffitt, \& Kramer, 1987; Bingham, 1995; Booth, Pinto, \& Bertenthal, 2002) and in studies on perception in bimanual coordination (Bingham, 2004; Bingham, Schmidt, \& Zaal, 1999; Bingham, Zaal, Shull, \& Collins, 2001; Wilson, Collins, \& Bingham, 2005a, 2005b; Zaal, Bingham, \& Schmidt, 2000).

More generally, however, events can be understood as spatial-temporal objects that exhibit characteristic shapes or forms, just as do more familiar spatial objects (Bingham, 1987, 1995; Bingham, Rosenblum, \& Schmidt, 1995; Runeson, 1974). Bingham (1995) formulated this in terms of trajectory forms. A trajectory consists of variations in the position and speed of a moving point. Trajectories exhibit path shapes and speed profiles along those paths. Such trajectory forms are different in different events. The oscillatory trajectory of a bouncing ball is quite different from that, for instance, of a manually oscillated hammer being used to hammer in a nail (Bingham et al., 1995). If trajectory forms are event-specific, they might be used to recognize events, just as the shapes of objects can be used to recognize trajectory forms.

Runeson (1983) argued that the physical dynamics responsible for generating the motions in an event produce unique kinematic (or motion) properties that enable observers who detect those motions to perceive corresponding dynamic properties of the events. Runeson (1983) developed this idea, which he called "KSD" (kinematic specification of dynamics), in the context of his work on perception of the mass of objects in collision events or amounts of weight in a human lifting event (Runeson, 1983; Runeson \& Frykholm, 1981, 1983). These are examples of the scaling problem in visual perception. How can the spatial-temporal optical pattern, which is angular (and therefore, only time-dimensioned) provide information about scale properties in events other than time (e.g., distance, size, mass, etc.)? Subsequent studies showed that the timing of readily identified gravitationally governed events (e.g., pendular events, balls rolling downhill, bouncing balls, falling water, walking dogs, etc.) provides information allowing the size and distance of objects in the events to be judged (Jokisch \& Troje, 2003; McConnell, Muchisky, \& Bingham, 1998; Pittenger, 1985, 1990;

E.A.Wickelgren, wickelgren@csus.edu 
Stappers \& Waller, 1993; Twardy \& Bingham, 2002; Watson, Banks, von Hafsten, \& Royden, 1992). The solution revealed by these studies is that the trajectories of visible points in an event are lawfully governed by the underlying event dynamics, which uniquely couple spatial and temporal properties so that one can provide information about the other.

Bingham (1995) extended Runeson's (1983) treatment to address the problem of event recognition. Bingham et al. (1995) showed that simple events consisting of a single moving point could be distinguished and recognized using the trajectory forms. Observers discriminated a freely bouncing object and a freely swinging object from those moved by hand. Furthermore, observers recognized each event - in particular, they correctly identified whether events were animate or inanimate. Other more complex events were similarly recognized (e.g., windblown objects; objects moving in stirred or splashed liquid; a kicked ball; a ball rolling downhill). Furthermore, the trajectory forms of many of these events were asymmetric and orientation-specific with respect to gravity. Bingham et al. (1995) found that when such trajectory forms were inverted, the events were no longer recognized. Similarly, observers have failed to recognize human walking when the event kinematics were inverted (Pavlova \& Sokolov, 2000; Sumi, 1984). Bingham et al. (1995) also tested the effect of changes in the relative orientation of observers and events by having inverted observers judge upright event kinematics; in this case, the events were correctly identified. These results show that the events are perceived relative to the generative dynamical context; that is, the downward gravitational force. The trajectory forms of events are specific to the dynamics that deterministically generate them. If the dynamics are specific to the type of event, trajectory forms can provide information about event types that would allow them to be recognized. Bingham et al. (1995) provided evidence that events are perceptually taxonimized in terms of the types of underlying generative dynamics.

These studies provided some evidence that human observers are sensitive to trajectory forms and can use them to recognize events. Some questions remain, however. First, trajectory forms can vary in two ways: The form of the speed profile along a given path of motion can change, and the form of the path of motion can change for a given speed profile. Are observers sensitive to both dimensions of variation of trajectory forms? Second, trajectory forms are properties of events that must be projected into optic flow to be detected by the visual system. What are the optic flow variables, and how are the two aspects of trajectory forms, path and speed, mapped into those variables? Third, the trajectory forms of events map into spatialtemporal optical patterns via perspective projections, so the forms are subject to perspective distortions, just as are the shapes of objects when projected into optical images. Does event recognition exhibit constancy (or view invariance); that is, does a given trajectory form allow the event to be recognized correctly when viewed from different 3-D perspectives? Previous studies have addressed some of these questions.
Muchisky and Bingham (2002) showed that, to recognize the event, adults can use the information in the speed profile of a nonlinear oscillator (an object oscillated along a straight path in a frontoparallel plane). Thresholds for both asymmetric (skew) and symmetric (kurtosis) variations in form were measured, as well as the ability to use the corresponding forms to reliably recognize the corresponding events. Thresholds were found to be comparable to those established for velocity discrimination, and observers were able to use the forms to recognize events.

Wickelgren and Bingham (2004) showed that people can use the information present in the speed profile of an event to recognize the same event when viewed from different perspectives. Participants viewed an object oscillating along a straight path in a frontoparallel plane with one of five different speed profiles and identified which event they were viewing. The same events were then viewed from the perspective of the straight path extending away in depth, so that the object moved toward and away from the observer. The perspective change incurred large perspective distortions in the trajectory forms. At the same time, the optical variables changed from rigid image translation to nonrigid image expansion and contraction; note that Johansson (1950) referred to these as common and relative motion components, respectively. Participants continued to be able to recognize the events despite these changes. When the path of motion was viewed from an oblique angle, the perspective distortions were carried simultaneously by both optical variables - that is, image translation and expansion/contraction - and the events were still recognized. Thus, human observers are able to detect speed profiles and use them to recognize events, despite projective distortions that can occur with changes in 3-D perspective.

Trajectory forms also vary in respect to path shape. The present studies were designed to investigate the use of path shape as information about event identity as well as the combination of path shape and speed profile. In Experiment 1, different events were created by varying the shape of the path along which an object traveled at constant speed. Observers became familiar with the events by viewing them from a perspective, which placed the paths in a frontoparallel plane. Subsequently, recognition was tested when events were viewed with the motion in a horizontal plane at eye level (thus, extending in depth) to determine the potential effects of perspective distortions. In Experiment 2, an object moved along a circular path with different speed profiles. Again, observers became familiar with the different events while viewing motion in a frontoparallel plane. Subsequently, recognition was tested while viewing motions in depth. Finally, in Experiment 3, both path shapes and speed profiles were varied. Five different path shapes were combined with 5 different speed profiles to create 25 different events. The ability to recognize the events in terms of the path shapes and speed profiles was tested when observers viewed the motions in depth so that viewing was subject to perspective distortions.

As shown in Appendix A, the speed profile and path shape of a trajectory form are specified in the optical 
flows. If $T_{\mathrm{o}}$ is the optical translation and $E_{\mathrm{o}}$ is the optical expansion/contraction component, the speed profile is specified by

$$
V(t)=D(t) * \sqrt{T_{\mathrm{o}}^{2}(t)+E_{\mathrm{o}}^{2}(t)},
$$

where $D(t)$ is the viewing distance. If viewing distance is large (that is, $D \gg \Delta D$, where $\Delta D=D_{\max }-D_{\min }$ over the course of the event), then $D(t) \approx D_{\mathrm{c}}$ (that is, $D$ constant). At closer distances, $D(t)=D_{\mathrm{c}}$ yields perspective distortions as discussed and investigated by Wickelgren and Bingham (2004). The path shape requires the direction of motion, $\phi(t)$, in addition to the speed. The direction is specified by $\phi(t)=\arctan \left[E_{\mathrm{o}}(t) / T_{\mathrm{o}}(t)\right]$. Given the availability of both speed and path in the optics, we expected that observers might perceive these events in terms of these two separable aspects of the form rather than as a single integral form; that is, they should be able to distinguish a set of speed profiles and a set of path shapes rather than the set of events composed as the product of these two sets.

\section{EXPERIMENT 1 Recognition of Path Shape From Different Perspectives}

Experiment 1 was designed to test how well people can recognize different path shapes when viewed from different perspectives. The speed profile was held constant and was, in fact, a constant speed. Observers were familiarized with 5 different path shapes viewed in a frontoparallel plane, then they were asked to identify these shapes when viewed in depth (that is, the plane containing the path, being horizontal at eye level, was viewed edge on).

\section{Method}

Participants. Twenty adults recruited from the introductory psychology subject pool participated in this study. Participants received course credit for their participation. As motivation, participants were told that those who had one of the two highest accuracies would receive a $\$ 20$ bonus.

Display generation. The events consisted of a ball that moved with a constant speed along one of five different path shapes (Figure 1): asymmetric, bimodal, circular, flower, and stretched. The paths were created using parametric equations for each shape. Since the object moved at a constant speed, the points along the path were equally spaced. Note that the asymmetric, bimodal, circular, and stretched path shapes mirrored the forms of the speed profiles used in Wickelgren and Bingham (2004). This enabled us to compare performance in the two cases - that is, with speed profiles and path shapes when tested separately and also when combined subsequently in Experiment 3.

For all five path shapes, we needed functions that ultimately formed a loop with no discernable beginning or ending. Whereas the circular- and flower-shaped paths were continuous functions, the asymmetric-, bimodal-, and stretched-shaped paths were created by piecing together portions of functions (pathwise continuous). To create the asymmetric path, hypotrochoid equations were used, as follows: $X=[(R-m R) * \cos (m t)]+\{h * \cos [t-(m t)]\}$ and $Y=$ $[(R-m R) * \sin (m t)]-\{h * \sin [t-(m t)]\}$, where $h$ is a constant that controls the size of the curve, $R$ is a constant that determines the number of involutes, and $m$ is a constant that determines the shape of the curve. They generate a triangular shape, as shown in Figure 1. To create the entire asymmetric path, the $X$ and $Y$ coordinates specifying this function were copied. The signs of the coordinates were changed to specify the bottom portion of the path and appended to the end of the original file specifying the top portion of the path.

The bimodal path, shown in Figure 1, was created similarly, but instead of a top and bottom portion, it had four quadrants, which were pieced together. The Lemniscate of Montferrier equations, $X=$ $a * \cos (t)$ and $Y=\left(a^{2} / 2 b\right) * \sin (2 t)$, were used to generate each quadrant, where $a$ is a constant that controls the size of the curve and $b$ is a constant that determines how peaked or rounded the curve is.

The circular path was created in its entirety using the parametric equations for a circle, where $a$ is a constant that controls the size of the curve: $X=a * \cos (t)$ and $Y=a * \sin (t)$.

The flower path was also created in its entirety using a version of the equations for a hypotrochoid, where $m$ is a constant controlling the shape of the curve, and $h$ controls the size: $X=[\cos (m t)]+$ $\{h[\cos (t-m t)]\}$ and $Y=[\sin (m t)]-\{h[\sin (t-m t)]\}$.

Similar to the bimodal path, the stretched path was created by splicing together four curves using the following equations, where $h$ is a constant that determines how peaked or rounded the curve is: $X=\{-[\cos (t)]\}^{\mathrm{h}}$ and $Y=[\sin (t)]^{\mathrm{h}}$. The sign of the equations determined the quadrant of the curve.

These events appeared in an upright view and a depth view. In the upright view, the event was projected so that the plane of the path was frontoparallel. Figure 2 shows how an event with the circular path appeared in the display. In this orientation, information about path shape was available from horizontal and vertical translation components; there were no changes in the image size of the round object. The diameter of the circular path was $12.5 \mathrm{~cm}$ and was centered on the computer screen. The left/right screen amplitude of the other four path shapes was $12.5 \mathrm{~cm}$, and the largest distance in the vertical dimension was $12.5 \mathrm{~cm}$. The duration of the events was $2 \mathrm{sec}$. The size of the object on the screen was $2.3 \mathrm{~cm}$. The observers were seated approximately $50 \mathrm{~cm}$ from the monitor, and the side-view events were simulated to be at the distance of the surface of the computer monitor. The visual angle for the displays was $13^{\circ}$, for an average optical velocity of approximately $13^{\circ} / \mathrm{sec}$. The events also appeared in a depth view, in which the plane of the path was perpendicular to the plane of the display and parallel to the line of sight. In this orientation, the object oscillated back and forth along a straight line on the screen (Figure 2). The image size of the object varied according to its distance as determined by the path in depth. The center of the circular path was simulated to be at the distance of the surface of the computer monitor, so that the object was traveling in front of, then behind, the plane of the computer monitor. The relationship of the image size to horizontal position provided information about path shape when the shape was viewed in depth. For each path shape, the closest simulated point of the path was $37.5 \mathrm{~cm}$ from the observer, and the farthest point of the path was $62.5 \mathrm{~cm}$. The same screen amplitude, object size, and period were used for the depth events with the same average optical velocity of $13 \%$ sec.

Design and Procedure. The experiment consisted of two phases, a training phase and a testing phase. The purpose of the training phase was to ensure that the observers could with reliable accuracy identify which path shape they were viewing in the upright condition. Before the training phase, the observers were shown the five path shapes in the upright condition, and were allowed to view them as many times as they wanted. Once they felt comfortable with the differences, they moved into the training phase of the experiment. In this phase, observers were presented with blocks of 25 trials each (the 5 path shapes each shown 5 times). The task of the observers was to identify which path they had just viewed: A, B, C, D, or E. After each identification, they were given feedback on their performance. If they were incorrect, they were told which display had been presented to them. After each block, the observers were told what their accuracy was for that block. If the criterion for accuracy was not reached, the observers performed another training block. The criterion for the training phase was an accuracy of $80 \%$ or better for each path shape $(80 \%$ accuracy for asymmetric path, $80 \%$ accuracy for bimodal path, etc.), for 2 blocks in a row. Once the criterion was achieved, the testing phase of the 
Asymmetric

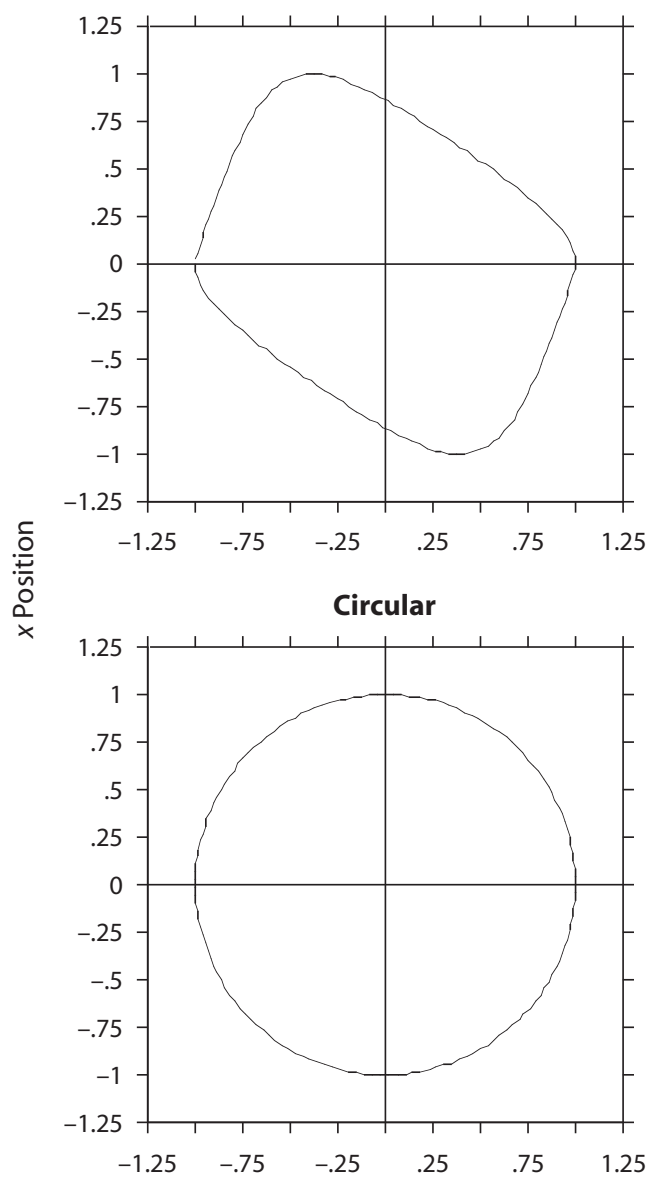

\section{Bimodal}

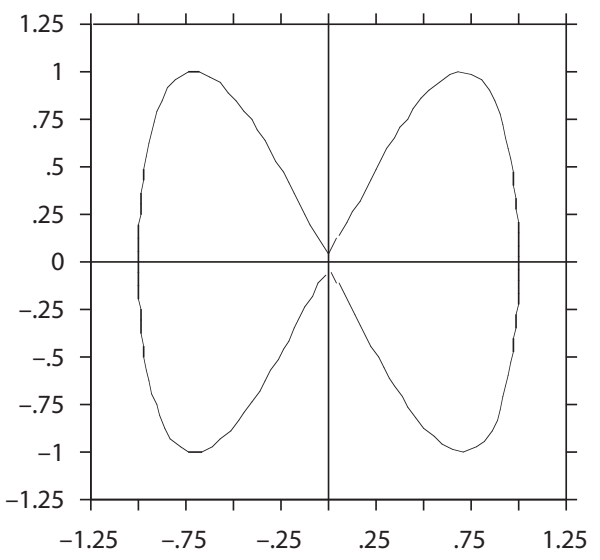

Flower

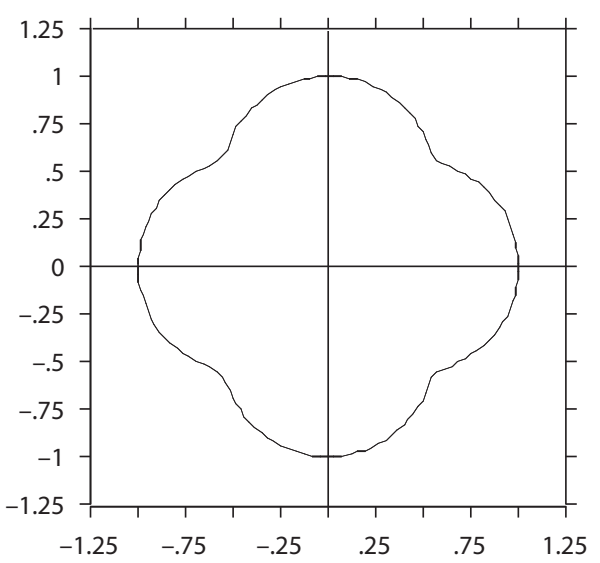

Stretched

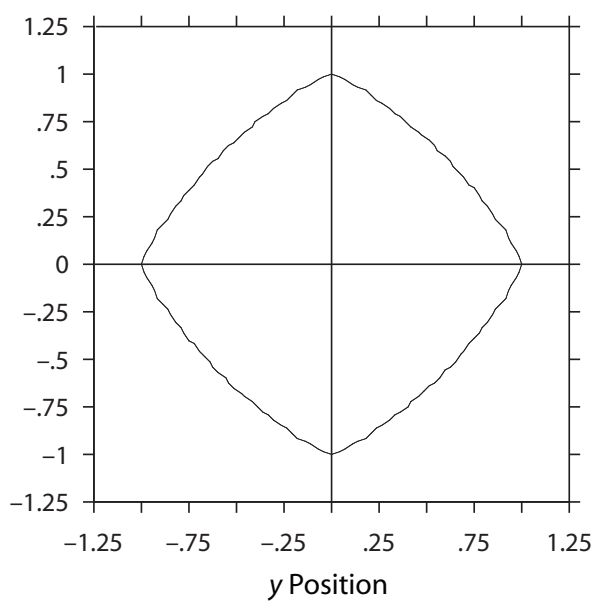

Figure 1. The five path shapes that were used in Experiment 1, plotting the $x$ position against the $y$ position. The units have been normalized.

experiment began. In this phase, the same path shapes were presented to the observers in 6 blocks of 25 trials each. However, the events were shown in the depth orientation, so that the image size of the circle changed according to its distance from the observer as it traveled back and forth across the screen. The task of the observer was the same as during the training phase: to identify which path shape was shown in depth. During the testing phase observers were not given feedback about their performance. 


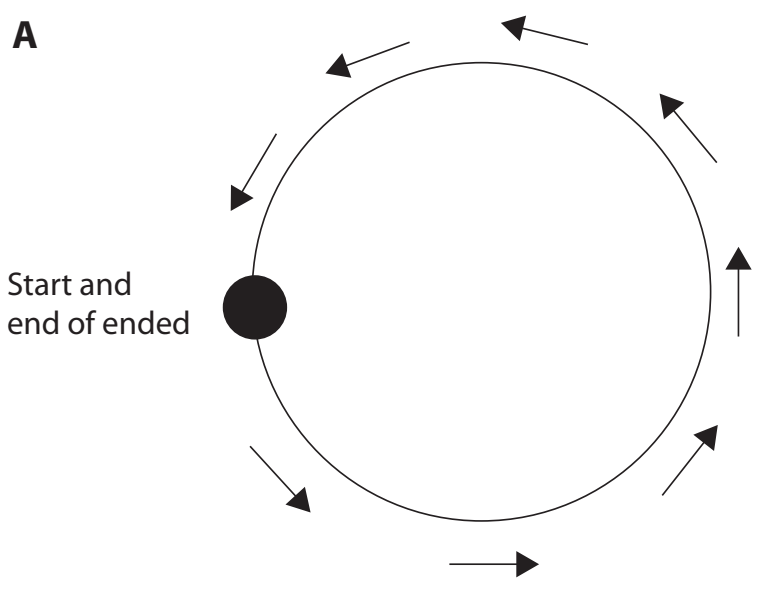

B

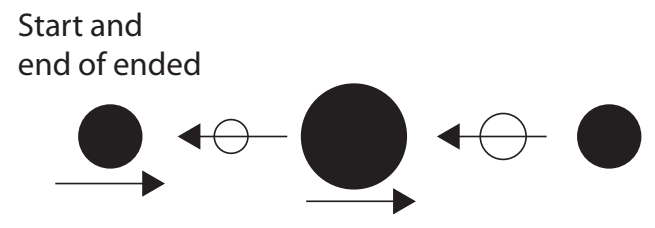

Figure 2. (A) The upright view orientation of the events during the training phase of Experiment 1. The event starts and stops at the same leftmost point on the path. (B) The depth-view orientation of the events during the testing phase of Experiment 1. Notice that there is no vertical component in the motion. The black circles show the change in image size that would occur as the ball moves around the circular path toward the observer, and the white circles represent the ball's image size on the back portion of the circular path as it returns to the left side of the display.

\section{Results and Discussion}

The combined response matrix for all 20 observers is shown in Appendix B. The first dependent measure calculated was percent correct, to assess how accurate identification was in the testing condition. During the training phase, observers had no difficulties identifying the path shapes; their mean terminal accuracy was $97.57 \%$ ( $S D=$ $2.53 \%$ ), and they only needed an average of 2.4 blocks to reach criterion (the minimum required was 2). The mean percent correct during the testing phase was $85.21 \%$ $(S D=7.41 \%)$, where chance performance would be $20 \%$. The observers were clearly not having much difficulty identifying the path shapes in the depth orientation.

The second dependent measure calculated was $d$-prime $\left(d^{\prime}\right)$. This was used to determine whether the observers tended to confuse any of the path shapes more than they did any of the others. First, $d^{\prime}$ were computed for each event compared with the other events combined (Table 1). For example, a $d^{\prime}$ was computed for the asymmetric path shape versus the other four path shapes combined, to see whether the asymmetric path was difficult to discriminate. Pairwise $d^{\prime}$ were also calculated for each combination of path shapes, to see whether any two displays were confused by participants (Table 2). A rule of thumb is that a $d^{\prime}$ above 1.0 indicates sensitivity to the difference in displays, whereas anything below 1.0 indicates that the two stimuli were confused (Macmillan \& Creelman, 1991). For example, the $d^{\prime}$ for the asymmetric event was 2.15 , indicating that observers had no difficulty discriminating the asymmetric path from the other path shapes. Overall, observers in this task did not appear to have any trouble identifying the path shapes viewed in depth (all $d^{\prime} \mathrm{s}>1.0$ ). To test whether any $d^{\prime}$ were significantly different from any other $d^{\prime}, 95 \%$ confidence intervals were computed for the difference in $d^{\prime}$ (Table 3). The results indicated that the bimodal and circular paths had the best performance (no significant difference), followed by the flower path, and the lowest discrimination performance was for the stretched and asymmetric path shapes (no significant difference). The results indicated that the comparison of the asymmetric path and stretched path had a significantly lower $d^{\prime}$ than did any other pairwise comparison. Although observers could still reliably discriminate between these two, their performance was

Table 1

Calculated $\boldsymbol{d}^{\prime}$ in Experiment 1 for Each Path Shape Paired With the Other Events Combined

\begin{tabular}{ll}
\hline \multicolumn{1}{c}{ Event Contrast } & $d^{\prime}$ \\
\hline Asymmetric vs. others & 2.15 \\
Bimodal vs. others & 3.87 \\
Circular vs. others & 3.73 \\
Flower vs. others & 3.34 \\
Stretched vs. others & 2.24 \\
Mean sensitivity & 3.06 \\
\hline
\end{tabular}

Table 2

Calculated $\boldsymbol{d}^{\prime}$ in Experiment 1 for Each Pairwise Comparison of Path Shape

\begin{tabular}{lc}
\hline Event Contrast & $d^{\prime}$ \\
\hline Asymmetric/bimodal & 5.39 \\
Asymmetric/constant & 3.44 \\
Asymmetric/harmonic & 3.74 \\
Asymmetric/stretched & 1.66 \\
Bimodal/constant & 6.27 \\
Bimodal/harmonic & 3.41 \\
Bimodal/stretched & 4.98 \\
Constant/harmonic & 5.06 \\
Constant/stretched & 3.91 \\
Harmonic/stretched & 4.36 \\
Mean sensitivity & 4.22 \\
\hline
\end{tabular}

Table 3

Ninety-Five Percent Confidence Intervals for Pairwise

Comparison of $d^{\prime}$ Values of Path Shapes in Experiment 1

\begin{tabular}{lc}
\hline \multicolumn{1}{c}{ Event Contrast } & $\begin{array}{c}d^{\prime} 95 \% \text { Confidence Interval } \\
\text { (Lower Bound, Upper Bound) }\end{array}$ \\
\hline Asymmetric/bimodal & $-1.97,-1.46^{*}$ \\
Asymmetric/circular & $-1.83,-1.32^{*}$ \\
Asymmetric/flower & $-1.41,-0.97^{*}$ \\
Asymmetric/stretched & $-0.28,0.10$ \\
Bimodal/circular & $-0.17,0.45$ \\
Bimodal/flower & $0.24,0.81^{*}$ \\
Bimodal/stretched & $1.37,1.88^{*}$ \\
Circular/flower & $0.10,0.67^{*}$ \\
Circular/stretched & $1.23,1.74^{*}$ \\
Flower/stretched & $0.87,1.33^{*}$ \\
\hline
\end{tabular}

*Significant at the .05 level. 
significantly worse than for other displays. This finding is notable, because Muchisky and Bingham (2002) found that the speed profile shapes that were most confused were the asymmetric and stretched speed profiles, both of which ex- hibit large variation in curvature, including sections of very low (nearly flat) curvature and sections of high curvature. These aspects seem to be highly salient, enough so to make the forms confusable despite the difference in symmetry.
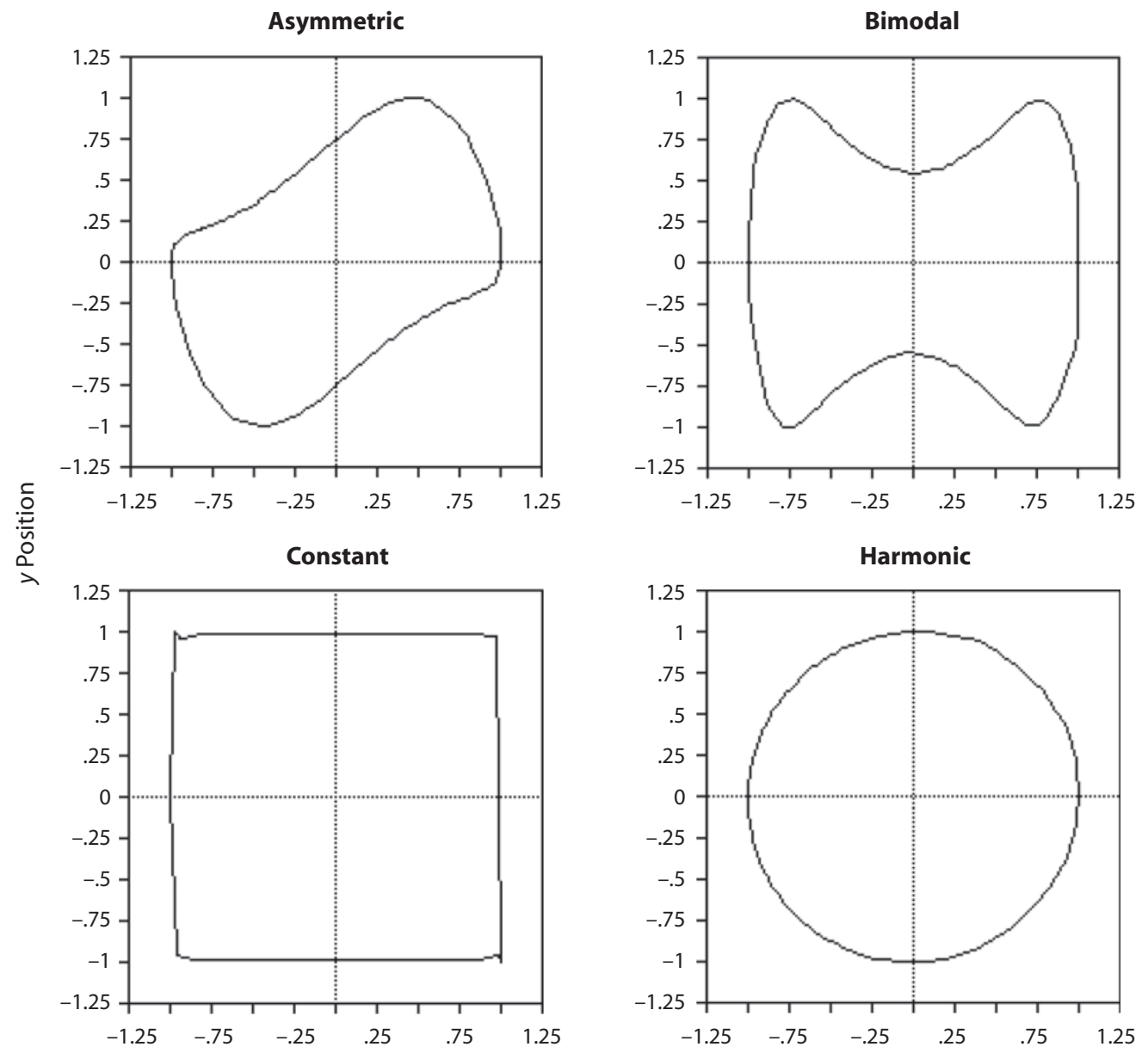

\section{Stretched}

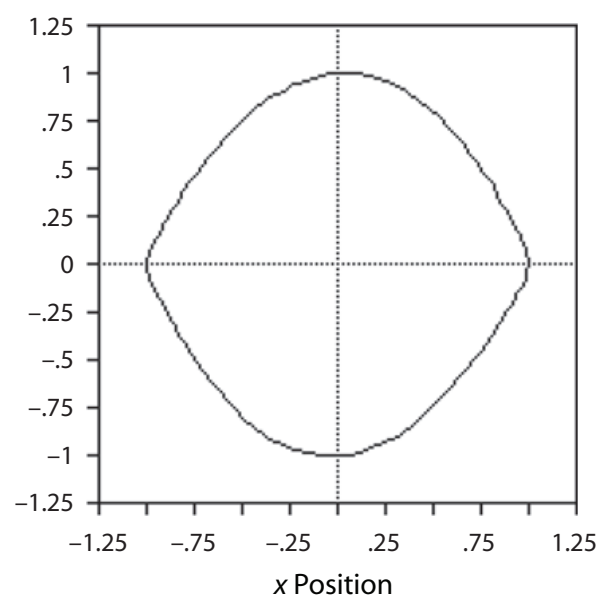

Figure 3. The five speed profiles that were mapped onto a circular path in Experiment 2. Velocity is plotted as a function of position along a straight lined path for each speed profile. The units have been normalized. 
In sum, the results of Experiment 1 showed that adults are very good at identifying path shapes viewed from different 3-D perspectives.

\section{EXPERIMENT 2 \\ Recognition of Speed Profiles Along a Curved Path}

Wickelgren and Bingham (2004) showed that adults are sensitive to speed profiles for events with a simple straight path; but paths of motion are rarely this simple. Experiment 2 was designed to determine whether people can identify speed profiles occurring along a more complex path viewed in depth - namely, a circle. Do people exhibit constancy in identifying such speed profiles from different 3-D perspectives?

\section{Method}

Participants. Sixteen adults participated in this study, and were each paid \$6. As motivation, participants were told that those who had one of the two highest accuracies would receive a $\$ 20$ bonus.

Design and Procedure. There were five event displays that varied in their speed profiles. The speed profiles of the events (asymmetric, bimodal, constant, harmonic, and stretched) were the same five forms as in Wickelgren and Bingham (2004) (Figure 3). The asymmetric form was generated using a van der Pol oscillator: $\ddot{X}=$ $-\lambda \dot{X}\left(X^{2}-1\right)-k X$, where $\lambda$ was a positive coefficient on the nonlinear damping term. As the value of $\lambda$ is increased, the peak speed of the event moves farther from the midpoint of the path, producing the characteristic asymmetric nature of the event. For each of the displays, we chose the same coefficients as those used in Wickelgren and Bingham. For the asymmetric velocity profile, $\lambda=1.75$ and $k=1.9$.

The bimodal speed profile was generated using the equation for a hard spring: $\ddot{X}=-c \cdot\left(X-k \cdot X^{3}\right)$, where $c$ is a constant controlling the period of the event, and $k$ is the nonlinearity determining the shape variation. For the bimodal speed profile, $c=1$ and $k=$ 2.5. By increasing $k$, the circular speed profile begins to flatten and eventually begins to dip in the middle, forming a bimodal profile with two peaks that occur at each end of the path.

In the constant speed profile, the object was constant in speed as it traveled along the path.

The harmonic velocity profile was created using a harmonic oscillator: $\ddot{X}=-k X$, where $k$ is the linear stiffness term and was set to 1.58 .

The final speed profile, stretched, was produced using the equation for a soft spring: $X=-c \cdot\left(X+k \cdot X^{3}\right)$. As with the equation for a hard spring, the constant $c$ controls the period of the event. As the nonlinearity, $k$, increases, the speed profile becomes increasingly peaked or stretched. For the stretched event, $c=7.52$ and $k=0.6$.

For each of these speed profiles, the object moved along a circular path (the same circular path used in Experiment 1). Speed profiles were mapped to paths by indexing points along the path in terms of proportional length along the path. The position values for each sample of a speed profile were normalized to a path length of 1 per cycle; that is, they were expressed as a proportion of the path length per cycle. The equations for the path shapes were reparameterized to path length, so the (proportional) path lengths for the speed profiles could be entered into the parameterized path shape equations to yield appropriate $x(t), y(t)$ coordinate values for each succeeding sample. The zero speed points of the speed profiles were mapped to the left and right endpoints on the screen.

The rest of the overall design and methods of this experiment were identical to those of Experiment 1. Participants performed a training phase where they were shown the events with the path in a frontoparallel plane and they received feedback on their identifications. Once they had achieved the $80 \%$ minimum accuracy, they proceeded to the testing phase where they viewed the displays in depth and received no feedback on their identifications.

\section{Results and Discussion}

The combined response matrix for all 16 observers is shown in Appendix C. Observers took more time than in Experiment 1 to learn the different events. Their mean terminal accuracy for the training phase was $90.07 \%$ ( $S D=$ $4.68 \%$ ), but they required an average of 3.94 blocks to reach criterion. The mean percent correct during the testing phase was $86.04 \%(S D=12.86 \%)$. Although it took them slightly longer to learn the different events, the participants did not have difficulty identifying the speed profiles in the depth orientation. Their performance was equal to that in Experiment 1.

As in Experiment 1, $d^{\prime}$ were first computed for each event compared with the other events combined (Table 4). All the $d^{\prime}$ were well above 1.0, indicating that participants were able to discriminate each of the displays from the others. To test if these $d^{\prime}$ were significantly different from each other, $95 \%$ confidence intervals were computed for the difference in $d^{\prime}$. There were significant differences among the $d^{\prime}$ for the events, with performance from highest to lowest as follows: constant, bimodal, harmonic, and the asymmetric and stretched speed profiles tied for lowest performance.

Pairwise $d^{\prime}$ were also calculated for each combination of path shapes to see whether any two particular displays were confused by participants (Table 5). Again, all the $d^{\prime}$ values were well above 1.0; overall in this task, therefore, observers exhibited constancy in their abilities to recognize speed profiles along a curved path viewed in depth. To test whether any $d^{\prime}$ were significantly different from any other $d^{\prime}, 95 \%$ confidence intervals were computed for the difference in $d^{\prime}$ (Table 6). The results indicated that the comparison of the asymmetric and stretched paths had a significantly lower $d^{\prime}$ than did any other pair-

Table 4

Calculated $d^{\prime}$ in Experiment 2 for Each Speed Profile, Paired With the Other Events Combined

\begin{tabular}{lc}
\hline \multicolumn{1}{c}{ Event Contrast } & $d^{\prime}$ \\
\hline Asymmetric vs. others & 2.41 \\
Bimodal vs. others & 3.54 \\
Circular vs. others & 4.71 \\
Flower vs. others & 2.80 \\
Stretched vs. others & 2.37 \\
Mean sensitivity & 3.17 \\
\hline
\end{tabular}

Table 5

Calculated $d^{\prime}$ in Experiment 2 for Each Pairwise Comparison of Speed Profiles

\begin{tabular}{lc}
\hline \multicolumn{1}{c}{ Event Contrast } & $d^{\prime}$ \\
\hline Asymmetric/bimodal & 4.96 \\
Asymmetric/constant & 6.07 \\
Asymmetric/harmonic & 3.34 \\
Asymmetric/stretched & 1.94 \\
Bimodal/constant & 4.31 \\
Bimodal/harmonic & 3.64 \\
Bimodal/stretched & 5.41 \\
Constant/harmonic & 5.58 \\
Constant/stretched & 6.10 \\
Harmonic/stretched & 2.89 \\
Mean sensitivity & 4.42 \\
\hline
\end{tabular}


wise comparison. Although observers could still reliably discriminate between these two, their performance was significantly worse than for other displays. These are the same two shapes most confused in Wickelgren and Bingham (2004) and in Muchisky and Bingham (2002), where speed profiles were tested, as well as in Experiment 1, where path shapes were tested. Clearly, these shapes are difficult to discriminate, however they are expressed. The overall conclusions from Experiment 2 are (1) adults can reliably recognize events with different speed profiles on a circular path, and (2) they exhibit constancy in identifying these events when they are viewed in depth.

\section{EXPERIMENT 3 \\ Recognition of Speed Profiles and Path Shapes}

Experiment 1 showed that adults can identify path shapes viewed from different perspectives when the speed profile is held constant; Experiment 2 showed that adults can identify the speed profile of an event on a curved path when the event is viewed from different perspectives. Experiment 3 was designed to test whether or not adults can simultaneously and separately identify path shapes and speed profiles when both are allowed to vary between events. It is possible that path shape and speed profile are detected as integral properties of trajectory forms, but the ability to detect these aspects separately would be more powerful and efficient.

We created a set of 25 different events by combining 5 different path shapes with 5 different speed profiles. Observers might perceive each event as a unique integral form; if so, they would have to become familiar with each of the 25 members of the set to be able to recognize the events. Nevertheless, we trained observers only with the 5 path shapes and 5 speed profiles. Observers were not familiarized with the 25 events composed of these respective subsets. The question was whether observers would nevertheless be able to recognize the events in terms of the path and speed forms. This was a strong test of the hypothesis that trajectory forms provide information for event recognition, because, strictly speaking, observers were not allowed to become familiar with the trajectory form for each event to be recognized. Furthermore, they were familiarized with path shapes and speed profiles

\section{Table 6}

Ninety-Five Percent Confidence Intervals for Pairwise Comparison of $\boldsymbol{d}^{\prime}$ Values of Speed Profiles in Experiment 2

\begin{tabular}{cc}
\hline Event Contrast & $\begin{array}{c}d^{\prime} 95 \% \text { Confidence Interval } \\
\text { (Lower Bound, Upper Bound) }\end{array}$ \\
\hline Asymmetric/bimodal & $-1.40,-0.87^{*}$ \\
Asymmetric/constant & $-2.76,-1.85^{*}$ \\
Asymmetric/harmonic & $-0.63,-0.16^{*}$ \\
Asymmetric/stretched & $-0.18,0.26$ \\
Bimodal/constant & $-1.64,-0.69^{*}$ \\
Bimodal/harmonic & $0.46,1.02^{*}$ \\
Bimodal/stretched & $0.91,1.44^{*}$ \\
Constant/harmonic & $1.44,2.36^{*}$ \\
Constant/stretched & $1.88,2.79^{*}$ \\
Harmonic/stretched & $0.20,0.67^{*}$ \\
\hline
\end{tabular}

${ }^{*}$ Significant at the .05 level. viewed from a different 3 -D perspective than the perspective from which recognition was tested. Another question addressed in this experiment, therefore, was whether observers would exhibit constancy of event recognition.

\section{Method}

Participants. Eight adults participated in this study and were each paid \$6. As motivation, participants were told that they would receive a $\$ 20$ bonus if they had one of the two highest accuracies.

Design and Procedure. In Experiment 3, each of the 5 speed profiles was mapped onto each of the path shapes, creating a total of 25 different events. For example, the asymmetric speed profile was mapped onto each of the 5 path shapes, the bimodal speed profile was then mapped onto each of the 5 path shapes, and so on. The displays were created using the same methodology as in Experiments 1 and 2 ; the speed profile determined the proportion of path length that the object traveled in each sample.

The design was similar to that in Experiments 1 and 2, with the following exceptions. First, the training portion of the experiment consisted of two separate training phases. The observers first performed a training phase to identify the path shape of the events. This training phase for path was identical to the training phase of Experiment 1. Observers viewed and identified each of the five path shapes with constant speed in the upright orientation and they were given feedback on their performance. Once the observers had achieved $80 \%$ accuracy or better for two consecutive blocks, they moved onto the next training phase, that for speed profile. The speed profile training phase was identical to the training phase in Experiment 2. Observers viewed each of the five velocity profiles on the circular path and made a judgment. They received feedback and needed to achieve at least an accuracy of $80 \%$ for two consecutive blocks before moving into the next phase of the experiment. After they had completed both training phases, observers were told that they would now see events that could be any combination of the five path shapes and speed profiles. Furthermore, they were told that they would be viewing the combined events in depth. The testing phase was identical to those in Experiments 1 and 2, except for the observers making two judgments instead of one. After viewing an event, they would first make a judgment identifying the speed profile, then one identifying the path shape. They were not given feedback during the testing phase.

\section{Results and Discussion}

The combined response matrices for the eight observers' judgments of path shapes and speed profiles are shown in Appendices D and E, respectively. As with the other experiments, we first calculated percent correct. The mean percent correct during the training phase for path shape was $97.41 \%(S D=5.33 \%)$, and the mean percent correct for the speed profile training phase was $91.67 \%$ $(S D=4.33 \%)$. It took the observers an average of 2.13 blocks to achieve the $80 \%$ accuracy minimum during the path-shape training, and an average of 3.25 blocks for the speed-profile training, which had also taken longer in Experiment 2 than had path-shape training in Experiment 1. Learning the speed profiles was more difficult.

The mean percent correct during the testing phase was $62.25 \%(S D=14.16 \%)$ for the path-shape judgments, and $51.17 \%(S D=15.46 \%)$ for the speed-profile judgments. In the previous two experiments in which these two tasks were performed separately, performance was approximately $85.21 \%$ for path shape and $86.04 \%$ for speed profile. The decrement in the performance levels could have been due to one or both of two circumstances: First, observers in this experiment had to remember and discriminate among twice as many forms as did observ- 
ers in the previous experiment; second, the two aspects of trajectory forms may have interacted so as to interfere with one another, especially in the context of the perspective distortions. Nevertheless, although performance was not as good in this experiment as in the previous experiments, these results indicate that observers were able to identify both the path shape and speed profile of an event, each separately, when the two aspects of trajectory form varied together and when the respective events were viewed in 3-D perspective.

To test for ability to discriminate between the events, we also calculated separate $d^{\prime}$ for the path shape and speedprofile judgments for each event compared with the other events combined (Table 7). All the $d^{\prime}$ for both path-shape and speed-profile judgments were above 1.0, except for the harmonic speed profile $\left(d^{\prime}=0.73\right)$, indicating that participants were able to discriminate all of the displays from the others, except when shown displays with a harmonic speed profile.

Pairwise $d^{\prime}$ were also calculated for each combination of path shape and each combination of speed profile, to see whether any two particular forms were confused by participants (Table 8). Again, all the $d^{\prime}$ values were above 1.0, except for the comparison of the asymmetric and stretched speed profiles $\left(d^{\prime}=0.94\right)$. A $95 \%$ confidence interval indicated that this was significantly worse than for the next best pairwise comparison (the bimodal/harmonic $d^{\prime}=$ 1.03) $(95 \% \mathrm{Ci}=-0.51,0.32)$. In Experiments 1 and 2 , the $d^{\prime}$ were lowest for this same combination of forms, although in the previous experiments observers were still discriminating between them. Despite this one comparison, it should be emphasized that participants were reliably discriminating between all of the path shapes and all but one of the speed profiles, when viewed entirely in depth. Overall, in this task, observers did exhibit perceptual constancy.

We also wanted to see whether observers were significantly better at identifying either the path shape or the speed profile in this experiment, so we computed the $95 \%$ confidence intervals comparing the mean $d^{\prime}$ value for the overall path comparisons (mean $d^{\prime}=1.74 ; S D=0.64$ ) and the overall speed profile comparisons (mean $d^{\prime}=$ $1.17 ; S D=0.38$ ) shown in Table 7. The confidence interval indicated that there was no difference between the $d^{\prime}$ values for the path shape and speed profile discriminations $(95 \% \mathrm{Ci}=-0.19,1.34)$. We also compared the $d^{\prime}$ values for the pairwise comparisons in Table 8. There was also no significant difference in the overall ability to discriminate the path shapes (mean $d^{\prime}=2.53 ; S D=.80$ ) and the ability to discriminate the speed profile shapes (mean $d^{\prime}=1.92$;

Table 7

Calculated $d^{\prime}$ in Experiment 3 for Each Path Shape and Speed Profile Paired With the Other Events Combined

\begin{tabular}{lll}
\hline \multicolumn{1}{c}{ Event Contrast } & $d^{\prime}$ for Path Shape & $d^{\prime}$ for Speed Profile \\
\hline Asymmetric vs. others & 1.11 & 1.05 \\
Bimodal vs. others & 2.62 & 1.03 \\
Circular vs. others & 1.73 & 1.76 \\
Flower or harmonic vs. others & 2.10 (flower) & 0.73 (harmonic) \\
Stretched vs. others & 1.18 & 1.29 \\
Mean sensitivity & 1.75 & 1.17 \\
\hline
\end{tabular}

Table 8

Calculated $d^{\prime}$ in Experiment 3 for

Each Pairwise Comparison of Path Shape and Speed Profiles

\begin{tabular}{lcc}
\multicolumn{1}{c}{ Event Contrast } & $\begin{array}{c}d^{\prime} \text { for } \\
\text { Path Shape }\end{array}$ & $\begin{array}{c}d^{\prime} \text { for } \\
\text { Speed Profile }\end{array}$ \\
\hline Asymmetric/bimodal & 2.69 & 1.93 \\
Asymmetric/constant & 1.73 & 3.20 \\
Asymmetric/flower (harmonic) & 2.27 & 1.29 \\
Asymmetric/stretched & 1.28 & 0.94 \\
Bimodal/constant & 3.52 & 1.44 \\
Bimodal/flower (harmonic) & 3.10 & 1.03 \\
Bimodal/stretched & 3.37 & 2.68 \\
Constant/flower (harmonic) & 3.07 & 1.72 \\
Constant/stretched & 1.48 & 3.69 \\
Flower (harmonic)/stretched & 2.75 & 1.30 \\
Mean sensitivity & 2.53 & 1.92 \\
\hline
\end{tabular}

$S D=.95)(95 \% \mathrm{Ci}=-0.22,1.43)$. Observers performed equally well on the two types of identifications.

\section{GENERAL DISCUSSION}

We investigated whether or not observers were able to use both aspects of trajectory forms to recognize events. Previous studies had shown that observers could use speed profiles viewed in either a frontoparallel plane or in 3-D perspective. We now investigated use of path shapes. In Experiment 1 , we found that observers could discriminate between and identify trajectory forms that varied only in path shape. When shown the same events in depth, observers could easily identify the events that had been seen in a frontoparallel plane. Next, we investigated the use of speed profiles on curvilinear paths in 3-D. In Experiment 2, observers were able to discriminate between different speed profiles in events with circular paths viewed in 3-D perspective.

Finally, we investigated whether observers were separately sensitive to either aspect of trajectory forms when the latter varied independently across events. Because information about speed and path are separately available in the optic flow, we expected that observers might be able detect their variations separately. Nevertheless, because it is possible that trajectory forms are detected as integral entities (meaning that observers would have to have become familiar with each of the 25 different events tested to be able to recognize the events), our experiments represented a strong test of the idea that trajectory forms might provide information used to recognize events. Instead, we found in Experiment 3 that observers were able to identify each of the two aspects of a trajectory form separately. With previous exposure and familiarization only with each of the 5 path shapes and the 5 speed profiles, they were subsequently able to recognize each of the 25 different events composed of these two parts. Furthermore, they were able to identify the events when viewed from different 3-D perspectives that entailed significant perspective distortions.

There is some evidence that observers might perceive trajectories as integral forms in which the two aspects, path shape and speed profile, are lawfully coordinated. Viviani (e.g., Lacquaniti, Terzuolo, \& Viviani, 1983; Viviani \& McCollum, 1983) performed a number of studies in which 
he investigated the kinematics of human limb movements including writing and drawing movements, reaching, and 3-D scribbling movements of the hand performed freely in the air. The results revealed an invariant scaling relation between the curvature of the path of movement and the speed along that path, such that the curvature and speed were inversely related by a $2 / 3$ power law. Subsequently, Viviani and Natale (1992) investigated the visual perception of such movements. They systematically varied the relation between path curvature and speed and asked observers to judge which speed profiles appeared constant. They found that trajectories exhibiting the $2 / 3$ scaling law were judged as constant speed trajectories. On the basis of this result, Viviani and Natale argued that human perception is especially attuned to such trajectories because they are characteristic of human limb movements.

Runeson (1974) had also investigated the perception of trajectories using phenomenological report measures. In that study, motion was always along a straight path in a frontoparallel plane. Observers were asked to draw the speed profiles for different trajectories including constant speed, acceleration to constant speed (which Runeson [1974] called "natural start"), constant acceleration, and constant deceleration. Runeson (1974) found that observers judged the "natural start" displays as constant speed. Bingham and Runeson (1983) subsequently replicated this study using the same displays but asking observers to draw the profile of the force that produced the movements. Observers drew the same graphs in both studies. But the important results were that (1) observers drew different graphs for each motion, which meant that they could discriminate among the motions; and (2) they consistently drew the same graph for each motion each time they judged it, meaning that they could recognize each of the motions. Bingham and Runeson concluded that no strong inferences should be drawn about the phenomenology, because observers produced the same reports when asked to judge velocity and force, respectively. Rather, Bingham and Runeson simply concluded that the trajectory forms could be detected and used to recognize events.

In Runeson (1974), a different trajectory type from that found in Viviani and Natale (1992) was judged as constant speed. This circumstance would also suggest that the phenomenology should not be strongly interpreted. Nevertheless, the Viviani and Natale studies indicate that some trajectories may be treated integrally by the visual system; this is also suggested by the orientation specificity found for trajectories of gravitational events. On the other hand, we found in the present study that path shape and speed profile can be used separately to recognize events. The results in Viviani and Natale also indicated that observers were able to discriminate differences in speed profiles occurring along a given path. The bottom line is that the visual system clearly can and does use trajectory forms to recognize events, and that a trajectory form is determined by both the path shape and the speed profile.

Finally, the problem of event recognition has also been investigated in machine vision. Often, the problem has been treated as a matter of predicting the next image in a sequence of images (Mann, Jepson, \& Siskind, 1997). Although Newtonian dynamics has been used in such efforts, the motion in events has not been used explicitly or directly. However, motions have been used in other efforts. In a number of papers, Davis has advocated an approach to the recognition of oscillatory events that applies trajectory templates to measured trajectories (e.g., Davis, 2001; Davis, Bobrick, \& Richards, 2000; Davis \& Richards, 2000). This work, however, requires that all motion occur in a frontoparallel plane; that is, it assumes that trajectories in 3-D space are the same as those in the 2-D optics. This, therefore, has little practical relevance to 3-D event recognition.

An approach in the machine vision literature that is closest to that described in the present paper is that of Rubin and Richards (1985), as well as that of Rao, Yilmaz, and Shah (2002). These investigators have explicitly addressed the problem of view invariance (that is, constancy) in the recognition of 3-D events. They characterize events in terms of certain qualitative properties of trajectories. They argue that discontinuities in speed and/or direction map into images in a view-invariant fashion and that, therefore, such qualitative information can be used to recognize events viewed from different perspectives. These investigators failed, however, to consider optic flow information about motion in events as described in the present article. Their analysis and approach was thus designed to grapple with an assumed loss of all information about 3-D trajectory components that lie in the depth direction - that is, parallel to the gaze axis. Naturally, in such an analysis, all metric properties of 3-D trajectories would be lost, as would be many other qualitative properties, such as actual 3-D path curvature. In contrast, our approach includes relevant analysis of optic flow information and thus does not assume this severe loss of information; indeed, our results show that successful event recognition is not limited to use of a spatial-temporal layout of discontinuities in trajectories. Instead, we found that smooth variations in both the path and speed of motion provide effective information for visual event recognition. Although some of the trajectory forms illustrated in Figures 1 and 3 did involve discontinuities in the changes of direction of speed of motion (e.g., the bimodal or flower path shapes, or the constant speed profile), a number of others did not (e.g., the asymmetric and circular path shapes, or most of the velocity profiles), and these were successfully discriminated and recognized. Furthermore, Muchisky and Bingham (2002) investigated discrimination of metric variations in trajectory forms and found thresholds equivalent to well established difference thresholds for speed discrimination (i.e., Weber fractions on the order of 5\%). Human observers are very sensitive to these dimensions of events.

The central insight contained in this research is the following: Understanding gained from the study of object recognition applies to event recognition. The problems and, presumably, the solutions are very closely related. It is the forms of events that allows them to be recognized, just as it is the forms of objects that makes them recognizable. The difference is that event recognition entails struc- 
ture existing over significant extents in time as well as in space. This will be the challenge in the future to theories about how the visual system detects and uses information to recognize events.

\section{AUTHOR NOTE}

We thank Nathaniel Blair for numerous valuable discussions about all aspects of this project, and Andrew Cohen for his generosity on helping to work out the best methods for display generation. Correspondence concerning this article should be addressed to E. A. Wickelgren, Department of Psychology, California State University, 6000 J St., Sacramento, CA 95819-6007 (e-mail: wickelgren@csus.edu).

\section{REFERENCES}

Bertenthal, B. I. (1996). Origins and early development of perception, action, and representation. Annual Review of Psychology, 47, 431-459.

Bertenthal, B. I., \& Pinto, J. (1993). Complementary processes in the perception and production of human movements. In E. Thelen \& L. B. Smith (Eds.), A dynamic systems approach to development: Applications (pp. 209-239). Cambridge, MA: MIT Press, Bradford Books.

Bertenthal, B. I., \& Pinto, J. (1994). Global processing of biological motions. Psychological Science, 5, 221-225.

Bertenthal, B. I., Proffitt, D. R., \& Cutting, J. E. (1984). Infant sensitivity to figural coherence in biomechanical motions. Journal of Experimental Child Psychology, 37, 213-230.

Bertenthal, B. I., Proffitt, D. R., \& Kramer, S. J. (1987). Perception of biomechanical motions by infants: Implementation of various processing constraints. Journal of Experimental Psychology: Human Perception \& Performance, 13, 577-585.

Bingham, G. P. (1987). Kinematic form and scaling: Further investigations on the visual perception of lifted weight. Journal of Experimental Psychology: Human Perception \& Performance, 13, 155-177.

Bingham, G. P. (1995). Dynamics and the problem of visual event recognition. In R. F. Port \& T. van Gelder (Eds.), Mind as motion: Dynamics, behavior and cognition (pp. 403-448). Cambridge, MA: MIT Press.

Bingham, G. P. (2004). Another timing variable composed of state variables: Phase perception and phase driven oscillators. In H. Hecht \& G. J. P. Savelsbergh (Eds.), Time-to-contact (Advances in Psychology, Vol. 135). Amsterdam: Elsevier.

Bingham, G. P., Rosenblum, L. D., \& Schmidt, R. C. (1995). Dynamics and the orientation of kinematic forms in visual event recognition. Journal of Experimental Psychology: Human Perception \& Performance, 21, 1473-1493.

Bingham, G. P., \& RunEson, S. (1983, October). On describing what is perceived: Seeing “velocity" versus seeing "push" in moving objects. Paper presented at the meeting of the International Society for Ecological Psychology, Nashville, TN.

Bingham, G. P., Schmidt, R. C., \& ZaAL, F. (1999). Visual perception of the relative phasing of human limb movements. Perception \& Psychophysics, 61, 246-258.

Bingham, G. P., ZaAl, F. T. J. M., Shull, J. A., \& Collins, D. R. (2001). The effect of frequency on visual perception of relative phase and phase variability. Experimental Brain Research, 136, 543-552.

Booth, A. E., Pinto, J., \& Bertenthal, B. I. (2002). Perception of the symmetrical patterning of human gait by infants. Developmental Psychology, 38, 554-563.

DAvis, J. W. (2001, November). Representing and recognizing human motion: From motion templates to movement categories. Paper presented at the Digital Human Modeling Workshop, International Conference on Intelligent Robots and Systems (IROS), Maui, HI.

DAVIS, J. [W.], BobRICK, A., \& RichaRds, W. (2000). Categorical representation and recognition of oscillatory motion patterns. IEEE Conference on Computer Vision \& Pattern Recognition, 2, 628-635.

DAVIS, J. W., \& RICHARDS, W. A. (2000). Relating categories of intentional animal motions (Tech. Rep. No. OSU-CISRC-11/00-TR25). Ohio State University: Department of Computer and Information Science.
Johansson, G. (1950). Configurations in event perception. Uppsala, Sweden: Almqvist \& Wiksell.

Jokisch, D., \& Troje, N. F. (2003). Biological motion as a cue for the perception of size. Journal of Vision, 3, 252-264.

Lacquaniti, F., Terzuolo, C., \& Viviani, P. (1983). The law relating the kinematic and figural aspects of drawing movements. Acta Psychologica, 54, 115-130.

Macmillan, N. A., \& Creelman, C. D. (1991). Detection theory: A user's guide. New York: Cambridge University Press.

Mann, R., JePson, A., \& Siskind, J. M. (1997). The computational perception of scene dynamics. ComputerVision \& Image Understanding, 65, 113-128.

McConnell, D. S., Muchisky, M. M., \& Bingham, G. P. (1998). The use of time and trajectory forms as visual information about spatial scale in events. Perception \& Psychophysics, 60, 1175-1187.

Muchisky, M. M., \& Bingham, G. P. (2002). Trajectory forms as a source of information about events. Perception \& Psychophysics, 64, 15-31.

Pavlova, M., \& SoKolov, A. (2000). Orientation specificity in biological motion perception. Perception \& Psychophysics, 62, 889-899.

Pittenger, J. B. (1985). Estimation of pendulum length from information in motion. Perception, 14, 247-256.

Pittenger, J. B. (1990). Detection of violations of the law of pendulum motion: Observers' sensitivity to the relation between period and length. Ecological Psychology, 2, 55-81.

RaO, C., Yilmaz, A., \& SHah, M. (2002). View-invariant representation and recognition of actions. International Journal of Computer Vision, 50, 203-226.

Rubin, J. M., \& RichaRds, W. A. (1985). Boundaries of visual motion (Tech. Rep. No. AIM-835). Cambridge, MA: Massachusetts Institute of Technology, Artificial Intelligence Laboratory.

Runeson, S. (1974). Constant velocity: Not perceived as such. Psychological Research, 37, 3-23.

RUNESON, S. (1983). On the visual perception of dynamic events. Uppsala, Sweden: Acta Universitatis Upsaliensis: Studia Psychologica Upsaliensia, 9, 1-56.

Runeson, S., \& Fryкholm, G. (1981). Visual perception of lifted weight. Journal of Experimental Psychology: Human Perception \& Performance, 7, 733-740.

Runeson, S., \& Frykholm, G. (1983). Kinematic specification of dynamics as an informational basis for person-and-action perception: Expectation, gender recognition, and deceptive intention. Journal of Experimental Psychology: General, 112, 585-615.

StapPERS, P. J., \& Waller, P. E. (1993). Using the free fall of objects under gravity for visual depth estimation. Bulletin of the Psychonomic Society, 31, 125-127.

SumI, S. (1984). Upside-down presentation of the Johansson moving light-spot pattern. Perception, 13, 283-286.

Twardy, C. R., \& Bingham, G. P. (2002). Causation, causal perception and conservation laws. Perception \& Psychophysics, 64, 956-968.

Viviani, P., \& McCollum, G. (1983). The relation between linear extent and velocity in drawing movements. Neuroscience, 10, 211-218.

Viviani, P., \& Natale, S. (1992). Biological movements look uniform: Evidence of motor-perceptual interactions. Journal of Experimental Psychology: Human Perception \& Performance, 18, 603-623.

Watson, J. S., Banks, M. S., von Hafsten, C., \& Royden, C. S. (1992). Gravity as a monocular cue for perception of absolute distance and/or absolute size. Perception, 21, 69-76.

Wickelgren, E. A., \& Bingham, G. P. (2004). Perspective distortion of trajectory forms and perceptual constancy in visual event identification. Perception \& Psychophysics, 66, 629-641.

Wilson, A. D., Collins, D. R., \& Bingham, G. P. (2005a). Human movement coordination implicates relative direction as the information for relative phase. Experimental Brain Research, 165, 351-361.

Wilson, A. D., Collins, D. R., \& Bingham, G. P. (2005b). Perceptual coupling in rhythmic movement coordination: Stable perception leads to stable action. Experimental Brain Research, 164, 517-528.

ZaAl, F., Bingham, G. P., \& Schmidt, R. C. (2000). Visual perception of mean relative phase and phase variability. Journal of Experimental Psychology: Human Perception \& Performance, 26, 1209-1220. 


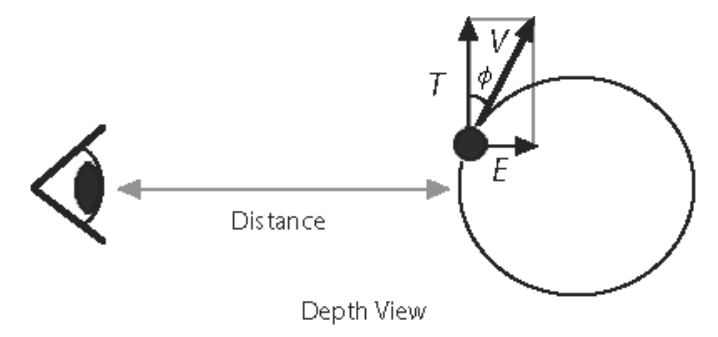

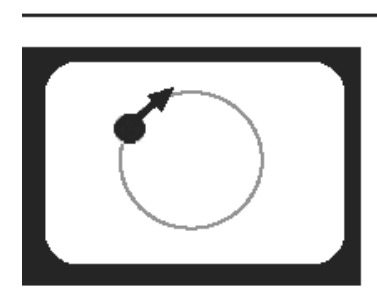

Upright View

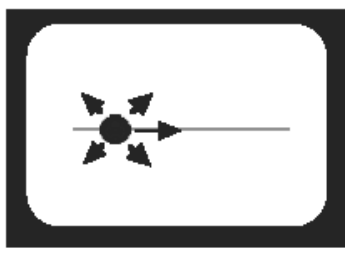

Depth View

Figure A1. The viewing geometry indicating the optic flow information for the path and speed profile of an event.

The viewing geometry is shown in Figure A1. A ball of size $S$ moves around a circular path at a velocity $V(t)$. The viewing distance is $D(t)$. The direction of motion is $\phi(t)$. The component of $V$ in a frontoparallel plane is

$$
T(t)=V(t) \cos [\phi(t)]
$$

and in the depth direction is

$$
E(t)=V(t) \sin [\phi(t)]
$$

The image size, $I(t)$, of the ball is

$$
I(t)=S / D(t)
$$

The image expansion rate, $I^{\prime}(t)$, is

$$
I^{\prime}(t)=I(t) * E(t) / D(t)
$$

We set the expansion component of the optics, $E_{o}(t)$, to $\tau(t)$. A $\tau(t)$ for the ball is computed as

$$
E_{\mathrm{o}}(t)=\tau(t)=I^{\prime}(t) / I(t)=E(t) / D(t) .
$$

The translation component, $T_{\mathrm{o}}(t)$, is

$$
T_{\mathrm{o}}(t)=T(t) / D(t)
$$

Then,

$$
\tan [\phi(t)]=E(t) / T(t)=E_{\mathrm{o}}(t) / T_{\mathrm{o}}(t),
$$

and $\phi(t)$ is specified optically as

$$
\phi(t)=\arctan \left[E_{\mathrm{o}}(t) / T_{\mathrm{o}}(t)\right] .
$$

To derive the optical specification of $V(t)$ :

$$
V(t)^{2}=T(t)^{2}+E(t)^{2}
$$

and

$$
V(t)=D(t) * \sqrt{T_{\mathrm{o}}(t)^{2}+E_{\mathrm{o}}(t)^{2}} .
$$

Assuming $D(t) \approx D c=\gamma$, then $V(t)$ is specified within an unknown parameter, $\gamma$ :

$$
V(t)=\gamma * \sqrt{T_{\mathrm{o}}(t)^{2}+E_{\mathrm{o}}(t)^{2}} .
$$


APPENDIX B

Confusion Matrices for Path Shape Identification in Experiment 1

\begin{tabular}{lrrrrr}
\hline \multirow{2}{*}{$\begin{array}{c}\text { Events } \\
\text { Presented }\end{array}$} & \multicolumn{5}{c}{ Observers' Responses } \\
\cline { 2 - 6 } \multicolumn{1}{c}{ Asym } & Bimo & Circ & Flow & Stre \\
\hline Asymmetric & 452 & 5 & 40 & 16 & 87 \\
Bimodal & 0 & 577 & 0 & 18 & 5 \\
Circular & 21 & 0 & 580 & 2 & 6 \\
Flower & 15 & 36 & 5 & 534 & 10 \\
Stretched & 143 & 2 & 25 & 5 & 425 \\
Total responses & 622 & 620 & 650 & 575 & 533 \\
\hline
\end{tabular}

APPENDIX C

Confusion Matrices for Identification of Speed Profiles Along a Circular Path in Experiment 2

\begin{tabular}{lrrrrr}
\hline \multirow{2}{*}{$\begin{array}{c}\text { Events } \\
\text { Presented }\end{array}$} & \multicolumn{5}{c}{ Observers' Responses } \\
\cline { 2 - 6 } & Asym & Bimo & Cons & Harm & Stre \\
\hline Asymmetric & 362 & 10 & 0 & 22 & 86 \\
Bimodal & 0 & 435 & 26 & 18 & 1 \\
Constant & 0 & 1 & 477 & 2 & 0 \\
Harmonic & 16 & 12 & 0 & 396 & 56 \\
Stretched & 66 & 2 & 0 & 17 & 395 \\
Total responses & 444 & 460 & 503 & 455 & 538 \\
\hline
\end{tabular}

APPENDIX D

Confusion Matrices for Path Shape Identification of Combined Events in Experiment 3

\begin{tabular}{lcrrrr}
\hline \multirow{2}{*}{$\begin{array}{c}\text { Events } \\
\text { Presented }\end{array}$} & \multicolumn{5}{c}{ Observers' Responses } \\
\cline { 2 - 6 } \multicolumn{1}{c}{ Asymmetric } & 97 & Bimo & Circ & Flow & Stre \\
Asymodal & 13 & 184 & 66 & 10 & 54 \\
Bimodar & 14 & 4 & 193 & 16 & 13 \\
Circular & 31 & 7 & 23 & 5 & 24 \\
Flower & 27 & 4 & 81 & 5 & 129 \\
Stretched & 182 & 212 & 377 & 186 & 243 \\
Total responses & & & & & \\
\hline
\end{tabular}

APPENDIX E

Confusion Matrices for Speed Profile Identification of Combined Events in Experiment 3

\begin{tabular}{lccccr}
\hline \multirow{2}{*}{$\begin{array}{c}\text { Events } \\
\text { Presented }\end{array}$} & \multicolumn{5}{c}{ Observers' Responses } \\
\cline { 2 - 6 } & Asym & Bimo & Cons & Harm & Stre \\
\hline Asymmetric & 105 & 31 & 7 & 34 & 63 \\
Bimodal & 13 & 97 & 46 & 70 & 14 \\
Constant & 8 & 31 & 158 & 39 & 4 \\
Harmonic & 40 & 27 & 25 & 106 & 42 \\
Stretched & 49 & 9 & 6 & 41 & 135 \\
Total responses & 215 & 195 & 242 & 290 & 258 \\
\hline
\end{tabular}

(Manuscript received September 8, 2006; revision accepted for publication August 21, 2007.) 